\title{
BMJ Open Prevalence and incidence of myalgic encephalomyelitis/chronic fatigue syndrome in Europe - the Euro-epiME study from the European network EUROMENE: a protocol for a systematic review
}

\author{
Fernando Estévez-López, , ${ }^{1,3}$ Jesus Castro-Marrero, ${ }^{4,5}$ Xia Wang, ${ }^{6}$ \\ Inger Johanne Bakken, ${ }^{7}$ Andrejs Ivanovs, ${ }^{8}$ Luis Nacul, ${ }^{9}$ Nuno Sepúlveda, ${ }^{10}$ \\ Elin B Strand, ${ }^{11}$ Derek Pheby, ${ }^{12}$ Jose Alegre, ${ }^{4}$ Carmen Scheibenbogen, ${ }^{13}$ \\ Evelina Shikova, ${ }^{14}$ Lorenzo Lorusso, ${ }^{15}$ Enrica Capelli, ${ }^{16}$ Slobodan Sekulic, ${ }^{17}$ \\ Eliana Lacerda, ${ }^{9}$ Modra Murovska, ${ }^{18}$ on behalf of the European Network on ME/ \\ CFS (EUROMENE)
}

To cite: Estévez-López F, Castro-Marrero J, Wang X, et al. Prevalence and incidence of myalgic encephalomyelitis/ chronic fatigue syndrome in Europe-the Euro-epiME study from the European network EUROMENE: a protocol for a systematic review. BMJ Open 2018;8:e020817. doi:10.1136/ bmjopen-2017-020817

\section{- Prepublication history for} this paper is available online. To view these files, please visit the journal online (http://dx.doi. org/10.1136/bmjopen-2017020817).

EL and MM contributed equally.

Received 20 December 2017 Revised 22 June 2018 Accepted 23 July 2018

Check for updates

(c) Author(s) (or their employer(s)) 2018. Re-use permitted under CC BY-NC. No commercial re-use. See rights and permissions. Published by BMJ.

For numbered affiliations see end of article.

Correspondence to Dr Fernando Estévez-López; f.estevez-lopez@uu.nl, festevez@ugr.es,

f.estevez-lopez@ulster.ac.uk

\section{ABSTRACT}

Introduction Myalgic encephalomyelitis/chronic fatigue syndrome (ME/CFS) is a chronic disease involving central nervous system and immune system disorders, as well as cardiovascular abnormalities. ME/CFS is characterised by severe chronic fatigue lasting for at least 6 months, including clinical symptoms such as tender cervical or axillary lymph nodes, muscle pain, joint pain without swelling or redness, post-exertional malaise for more than 24 hours and unrefreshing sleep. Studies on the epidemiology of ME/CFS in Europe only include single countries and, therefore, the prevalence and incidence of ME/CFS in Europe (as a whole) is unknown. One of the purposes of the European Network on ME/CFS (EUROMENE; European Union-funded COST Action; Reference number: 15111) is to address this gap in knowledge. We will systematically review the literature reporting figures from European countries to provide a robust summary and identify new challenges.

Methods and analysis We will systematically search the literature databases Scopus, PubMed and Web of Science for studies published in the last 10 years (ie, after 2007). No language restriction will be applied. Two independent reviewers will search, screen and select studies as well as extract data about their main characteristics and evaluate their methodological and reporting quality. When disagreements emerge, the reviewers will discuss to reach a consensus. We plan to produce a narrative summary of our findings as we anticipate that studies are scarce and heterogeneous. The possibility of performing metaanalyses will be discussed in a EUROMENE meeting. Ethics and dissemination Ethical approval is not required as only publicly available data will be included. Findings will be described in EUROMENE reports, published in peer-reviewed journal(s) and presented at conferences. The findings will be also communicated to policy-makers,

\section{Strengths and limitations of this study}

- The main strengths of this protocol rely on its carefully designed search strategy, inclusion and exclusion criteria, and time-span coverage.

- The search strategy will address the potential EU studies published in non-English national languages, and we will include currently accepted myalgic encephalomyelitis/chronic fatigue syndrome (ME/ CFS) case definitions, to minimise selection bias.

- Studies based on self-report will be excluded, and the search time of 10 years will enable us to picture the ME/CFS occurrence in Europe.

- The European Network on ME/CFS (EUROMENE), a network of established researchers on ME/CFS, will conduct the proposed systematic review which can increase credibility and reliability of the findings.

- A potential limitation of this review may be a small number of studies available and their potential high heterogeneity.

healthcare providers, people with ME/CFS and other sections of society through regular channels including the mass-media.

PROSPERO registration number CRD42017078688

\section{INTRODUCTION}

Myalgic encephalomyelitis/chronic fatigue syndrome (ME/CFS) has heterogeneous clinical features and is characterised by severe fatigue lasting for at least 6 months that is medically unexplained and not relieved by resting. ${ }^{1}$ Consequently, ME/CFS often 
imposes a huge burden on daily life with negative impacts on health-related quality of life, labour status, and social and familial relationships. ${ }^{2-4}$ Societal disbeliefs may add to the burden for people with $\mathrm{ME} / \mathrm{CFS} .^{35}$

For several reasons, ME/CFS is a challenge for scientists. First, to date, the features of ME/CFS have been poorly defined. For instance, an intense physical discomfort (ie, malaise) along with flares of ME/CFS symptoms (lasting for longer than 24 hours) is observed after minimum physical exertion. ${ }^{6}$ However, whether post-exertional malaise is a distinctive feature of ME/CFS is not yet fully agreed. ${ }^{7}$ Second, related to the lack of consensus on its defining features, a large number of disparate criteria for the diagnosis of ME/CFS are currently used worldwide. ${ }^{8}$ Indeed, the most common scenario is to diagnose ME/CFS after exclusion of other diseases. ${ }^{4-13}$ Third, as different diagnostic criteria are used, ME/ CFS prevalence and incidence figures are highly variable across studies. For instance, within an Icelandic study, ${ }^{14}$ the prevalence of ME/CFS ranged from $0 \%$ to $5 \%$ by means of the Lloyd $e t a l^{15}$ or Holmes $e t a l^{16}$ criteria, respectively.

To address the above-mentioned caveats and others, it is imperative to understand ME/CFS comprehensively. With this purpose, the European Network on ME/CFS (EUROMENE) was established. This (EU-funded COST Action; Reference number: 15111) multidisciplinary network involves patients, stakeholders, researchers, clinicians and industry. We intend to align ME/CFS research within the established landscape of European biomedical research by developing additional proposals to the new H2020 and further Framework programme collaborative research projects. Further information about the EUROMENE network is available at http://www.cost.eu/ COST_Actions/ca/CA15111 and http://www.euromene. $\mathrm{eu} /$.

EUROMENE consists of six closely coordinated working groups. Working group 1 focuses on the epidemiology of ME/CFS and leads the European Epidemiological Study for ME/CFS (Euro-EpiME study). One specific aim of this study is to estimate the prevalence and incidence of ME/CFS in Europe. As a first step, we will systematically review the available literature from European countries in order to provide a robust summary and identify new challenges in the field. It seems likely that more data on the prevalence and incidence of ME/CFS will be needed, both for Europe as a whole and within the European countries. Previously, systematic reviews have been conducted including studies from many parts of the world. ${ }^{17-19}$ However, these previous reviews: (1) were conducted more than 5 years ago, ${ }^{20}$ (2) did not report the incidence of ME/CFS and (3) did not include children or adolescents.

\section{Objective}

We aim to conduct a systematic review and, if possible, meta-analyses to determine the prevalence and incidence of ME/CFS in Europe.

\section{Review question}

1. What is the prevalence of ME/CFS in Europe?

2. What is the incidence of ME/CFS in Europe?

\section{METHODS}

\section{Inclusion criteria}

1. Studies reporting either the prevalence or incidence of ME/CFS, including any of the following clinical diagnostic criteria: CDC-1994, ${ }^{9}$ Canadian Consensus Criteria, ${ }^{1}$ London Criteria, ${ }^{21}$ International Consensus Criteria $^{10}$ or Institute of Medicine criteria, ${ }^{22}$ irrespective of age groups.

2. Studies from European countries.

3. Studies in community or primary care settings.

\section{Exclusion criteria}

1. Studies without primary data (eg, reviews).

2. Studies conducted in biased samples (eg, vaccines, virus infection, veterans).

3. Studies based on self-report of the diagnosis of ME/ CFS.

4. Studies with an inappropriate case definition (eg, CFSlike illness or other clinical criteria, such as the Oxford criteria due to lack of specificity).

5. Duplicate reports. When populations are overlapping, the study with the largest sample size will be included.

6. Studies published more than 10 years ago (ie, before 2008).

No language restriction will be applied.

\section{Search strategy for identifying relevant studies}

The search strategy will consist of two stages: a primary systematic literature search on three electronic databases and a complementary search.

The primary systematic literature search on electronic databases Two independent reviewers (FE-L and JC-M) will perform a primary electronic search in PubMed, Scopus and Web

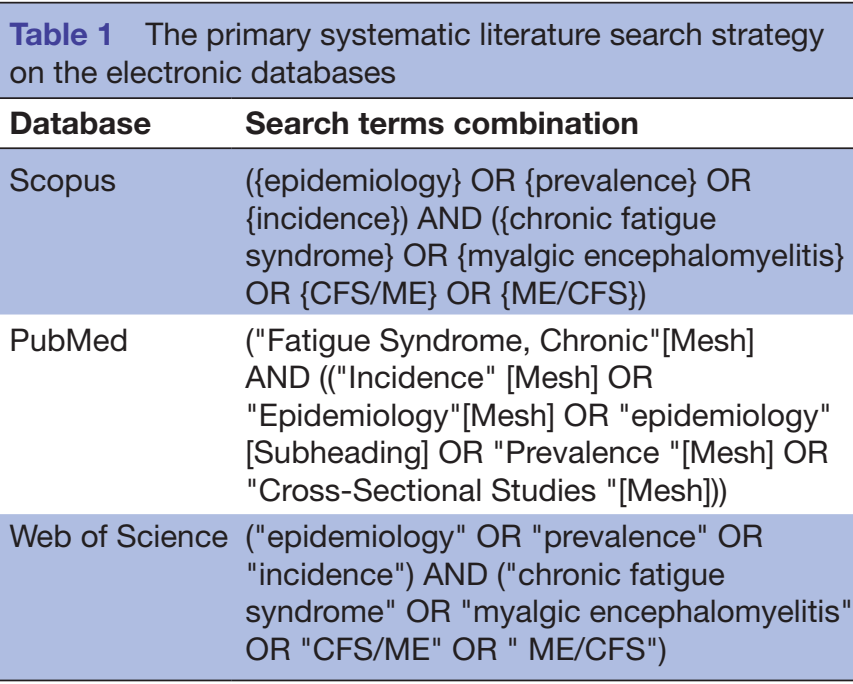

Mesh, medical subject headings. 
of Science on 9 January 2018. Table 1 shows the search strategy.

\section{The complementary search}

We will conduct a twofold complementary search as follows: first, we will perform a backward (by checking reference lists) and forward (by checking citations) search of the works included in the present review; and second, grey literature will be addressed by contactingvia email-all the members of EUROMENE to provide, if available, prevalence rates, incidence rates or both of $\mathrm{ME} / \mathrm{CFS}$ in their countries according to national registers, publications in their own languages or any other publicly accessible source.

\section{Selection of studies for inclusion to the review}

Two independent researchers (FE-L and JC-M) will screen records retrieved by the electronic search by titles/ abstracts or full text of works for identifying potential studies and their suitability. When disagreements emerge between the two independent researchers, consensus will be obtained through discussion or when required, the opinion of a third researcher (IJB) will be considered.

\section{Assessment of methodological quality and reporting of data}

The methodological quality of the eligible studies will be evaluated with the Joanna Briggs Institute-Checklist for Prevalence Studies. ${ }^{23}$ Before applying it, six members of the research team (ie, FE-L, LN, JA, SS, EL and MM) will develop an agreed appraisal of the tool. This appraisal will be published with the review as supplementary information. The reporting quality of the eligible studies will be evaluated using the Strengthening the Reporting of Observational Studies in Epidemiology checklist. ${ }^{24}$ Two independent researchers (ie, AI and XW) will evaluate the methodological and reporting quality of the included works. When controversies emerge, studies will be discussed with two other members of the team in order to reach a consensus (ie, EBS and DP for methodological and reporting quality, respectively). The quality assessment will be considered when discussing the findings.

\section{Data extraction and management}

To manage the retrieved records from the electronic search, we will use the Mendeley Desktop. Two independent researchers (FE-L and AI) will extract the following relevant data from the included studies: reference (authors and year of publication), country (city or region when relevant), design (ie, general population online survey), total sample size ( $\mathrm{n}$ and $\%$ of women, $\mathrm{n}$ and $\%$ of migrants), age range, setting (eg, primary care), case definition (ie, diagnosis criteria), dates of data collection, overall prevalence and/or incidence and prevalence and/or incidence by gender and age groups (when available). When discrepancies emerge in the coding between the two researchers' results, these will be discussed with another members of the team (ie, NS) to reach a consensus.

\section{Data synthesis and analysis}

We anticipate that studies on the prevalence and incidence of ME/CFS in Europe will prove to be scarce and heterogeneous. The preliminary findings of the review will be presented in a EUROMENE meeting (ie, September 2018, in London, UK) where we will discuss the appropriateness of performing meta-analyses.

A narrative (descriptive) synthesis is planned if meta-analyses are not feasible. We will pay special attention to possible factors related to heterogeneity of the findings in order to find patterns that should be considered in future research. For instance, we will discuss whether the prevalence or incidence of ME/CFS differ according to the case definition used to examine the figures. Attention will be also paid to the characteristics of the studied populations (eg, age group, gender).

We will undertake meta-analyses only where pooling of quantitative data is possible. The meta regression analysis will be performed to investigate the sources of heterogeneity of any ME/CFS pooled prevalence and incidence estimate. The $\mathrm{I}^{2}$ statistic will be used to investigate the heterogeneity. $\mathrm{I}^{2}$ of $25 \%, 50 \%$ and $75 \%$ will be appraised as low, moderate and high, respectively. ${ }^{25}$

\section{Presentation and reporting of results}

We will report the findings of the present literature review in accordance with the Preferred Reporting Items for Systematic Reviews and Meta-Analyses statement. ${ }^{26}$ A flow diagram (figure 1) will illustrate the process of study selection from retrieved records to included studies. For transparency purposes, supplementary files will show which studies were excluded at every stage of the review. If the present protocol needs amendments, they will be publicly available along with their rationale on the EUROMENE website (http://www.euromene.eu/).

In accordance with the data extraction, a table will show the main characteristics of the studies included. Information on the quality of the methodology and reporting of the studies will also be available.

If meta-analyses are performed, we plan using comprehensive meta-analysis to combine data of prevalence or incidence from different studies to estimate the mean effect. We will select an appropriate model in terms of studies that we include and calculate pooled ME/CFS prevalence and incidence. We will compare the results of random-effects and fixed-effect meta-analyses in sensitivity analyses.

\section{Ethics and dissemination}

The findings of this systematic review will address a specific aim of the European network EUROMENE (ie, to summarise the available data of the prevalence and incidence of ME/CFS in Europe). The findings will be included in EUROMENE reports published in paper(s) in peer-reviewed journal(s) and presented at conferences and meetings.

The findings of the present systematic review will be widely communicated to society using mass media 


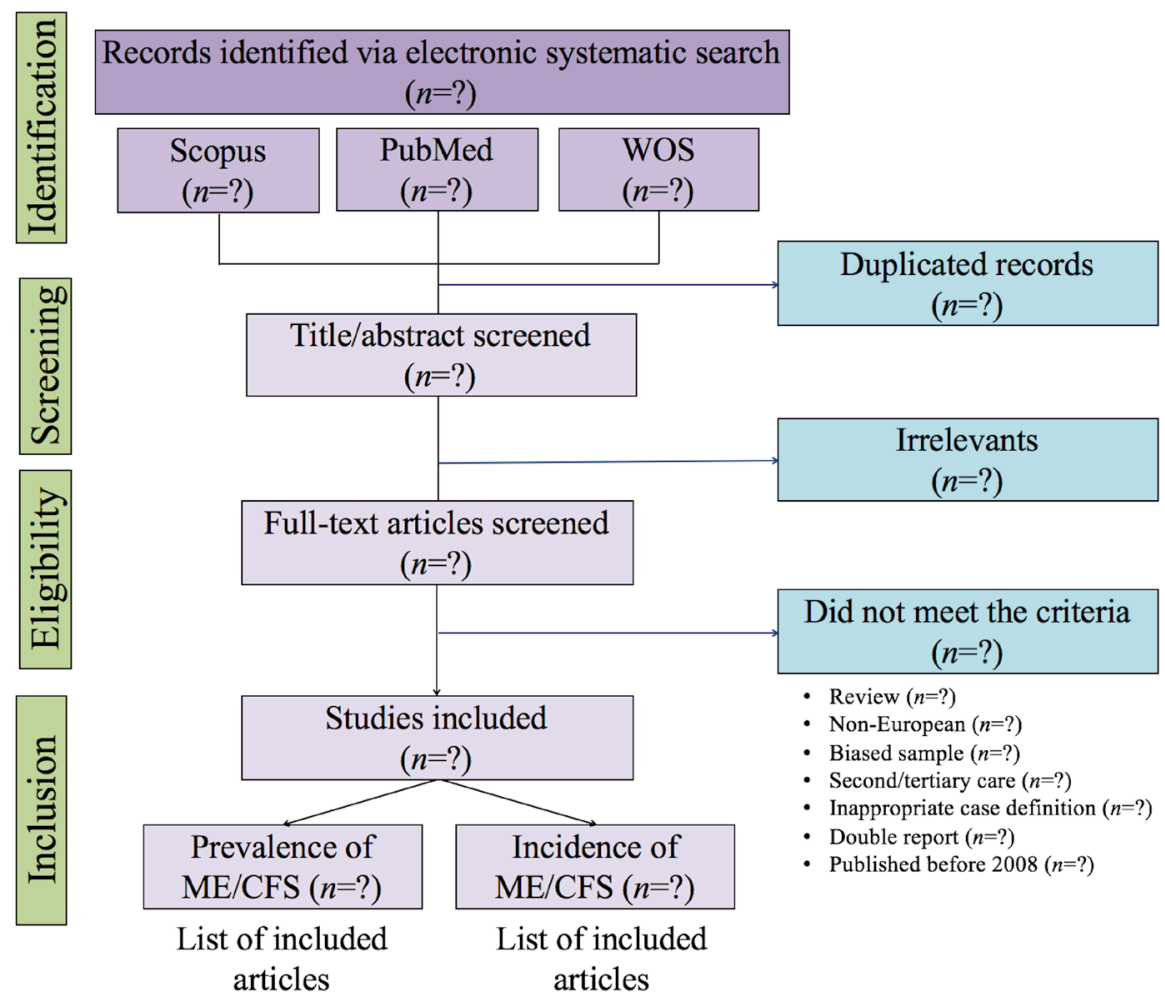

Figure 1 Flow diagram for study selection. ME/CFS, myalgic encephalomyelitis/chronic fatigue syndrome; WOS, Web of Science.

(eg, interviews on radio, newspaper, television and the internet). Since our findings may have an impact on policy and healthcare practice, we will also present them to policy-makers and healthcare providers.

\section{Patient and public involvement}

EUROMENE is multidisciplinary network cooperating with patient organisations via Web platform. Patient organisations are benefiting from dedicated events, dedicated printed media and interaction through social media. We will present our findings to patients with $\mathrm{ME} /$ CFS (eg, by direct communication with representative patient organisations and by giving talks to local associations of people with ME/CFS). General public will be reached through the COST Action website, oral presentations and interviews.

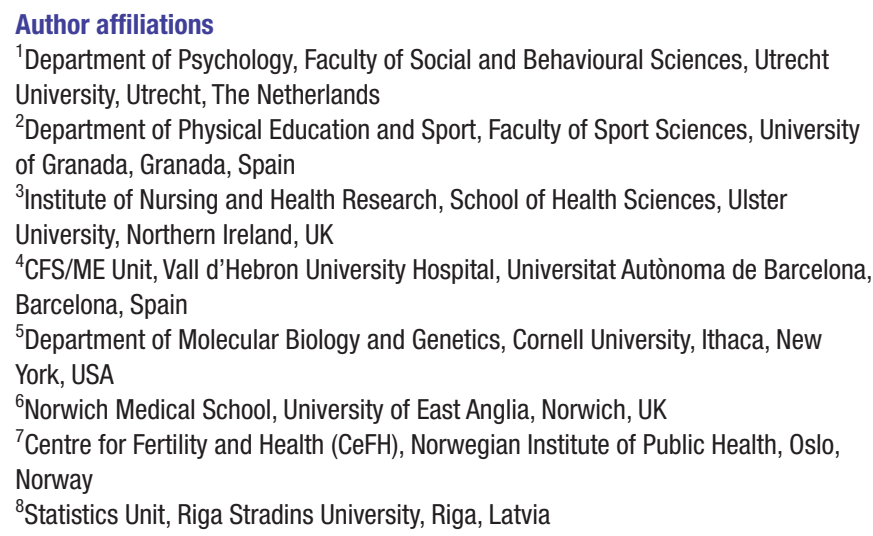

${ }^{5}$ Department of Molecular Biology and Genetics, Cornell University, Ithaca, New York, USA

${ }^{6}$ Norwich Medical School, University of East Anglia, Norwich, UK

${ }^{7}$ Centre for Fertility and Health (CeFH), Norwegian Institute of Public Health, Oslo, Norway

${ }^{8}$ Statistics Unit, Riga Stradins University, Riga, Latvia

${ }^{9}$ Department of Clinical Research, Faculty of Infectious \& Tropical Disease, London School of Hygiene \& Tropical Medicine, London, UK

${ }^{10}$ Centre of Statistics and Its Applications, University of Lisbon, Lisbon, Portugal

${ }^{11}$ National Advisory Unit on CFS/ME, Oslo University Hospital, Oslo, Norway

${ }^{12}$ Faculty of Health and Society, Buckinghamshire New University, High Wycombe, UK

${ }^{13}$ Institute for Medical Immunology, Charité-Universitätsmedizin Berlin, Berlin, Germany

${ }^{14}$ Department of Virology, National Center of Infectious and Parasitic Diseases, Sofia, Bulgaria

${ }^{15}$ Neurology Department, ASST-Lecco, Merate, Italy

${ }^{16}$ Department of Earth and Environmental Sciences and Centre for Health

Technologies, University of Pavia, Pavia, Italy

${ }^{17}$ Department of Neurology, Medical Faculty Novi Sad, University of Novi Sad, Novi Sad, Serbia

${ }^{18}$ August Kirchenstein Institute of Microbiology and Virology, Riga Stradins University, Riga, Latvia

Acknowledgements The present protocol of systematic review was agreed in a EUROMENE meeting in Belgrade (Serbia) on 7 September 2017. We thank all the participants for their active participation and valuable suggestions.

Contributors FE-L, JC-M, XW, EL and MM designed the protocol. FE-L drafted the manuscript. FE-L, JC-M, XW, IJB, Al, LCN, NS, EBS, DP, JA, CS, ES-L, LL, EC, SS,

$E L$ and $M M$ revised and approved the final version of the manuscript. MM and EL are the chair and vice chair of the EUROMENE action, respectively. FE-L will be the guarantor of the review.

Funding This work was supported by the COST (Action CA 15111: The European Network on Myalgic Encephalomyelitis/Chronic Fatigue Syndrome (EUROMENE), the Spanish Ministry of Economy and Competitiveness [BES-2014-067612 to F.E.-L.], the Bulgarian National Science Fund [ДКОСТ 01/9 to E.S.].

Disclaimer The funders of the present study did not have any role in the design, decision to publish or preparation of the protocol.

Competing interests None declared.

Patient consent Not required. 
Ethics approval As systematic reviews use publicly available data, no formal ethical review and approval is needed.

Provenance and peer review Not commissioned; externally peer reviewed.

Open access This is an open access article distributed in accordance with the Creative Commons Attribution Non Commercial (CC BY-NC 4.0) license, which permits others to distribute, remix, adapt, build upon this work non-commercially, and license their derivative works on different terms, provided the original work is properly cited, appropriate credit is given, any changes made indicated, and the use is non-commercial. See: http://creativecommons.org/licenses/by-nc/4.0/.

\section{REFERENCES}

1. Carruthers B, Jain AK, De Meirleir KL, et al. Myalgic encephalomelitis/chronic fatigue syndromw: clinical working case definiton, diagnostic and treatment protocols. J Chronic Fatigue Syndome 2003;11:7-115.

2. Lowry TJ, Pakenham KI. Health-related quality of life in chronic fatigue syndrome: predictors of physical functioning and psychological distress. Psychol Health Med 2008;13:222-38.

3. de Carvalho Leite JC, de L Drachler M, Killett A, et al. Social support needs for equity in health and social care: a thematic analysis of experiences of people with chronic fatigue syndrome/myalgic encephalomyelitis. Int J Equity Health 2011;10:46.

4. Nacul LC, Lacerda EM, Campion P, et al. The functional status and well being of people with myalgic encephalomyelitis/chronic fatigue syndrome and their carers. BMC Public Health 2011;11:402.

5. Raine R, Carter S, Sensky T, et al. General practitioners' perceptions of chronic fatigue syndrome and beliefs about its management, compared with irritable bowel syndrome: qualitative study. BMJ 2004;328:1354-7.

6. McManimen SL, Sunnquist ML, Jason LA. Deconstructing postexertional malaise: An exploratory factor analysis. J Health Psychol 2 016;1359105316664139:135910531666413.

7. Jason LA, Evans M, So S, et al. Problems in defining post-exertional malaise. J Prev Interv Community 2015;43:20-31.

8. Nacul L, Lacerda EM, Kingdon CC, et al. How have selection bias and disease misclassification undermined the validity of myalgic encephalomyelitis/chronic fatigue syndrome studies? J Health Psychol 2017:135910531769580.

9. Fukuda K, Straus SE, Hickie I, et al. The chronic fatigue syndrome: a comprehensive approach to its definition and study. International Chronic Fatigue Syndrome Study Group. Ann Intern Med 1994:121:953-9.

10. Carruthers BM, van de Sande MI, De Meirleir KL, et al. Myalgic encephalomyelitis: international consensus criteria. J Intern Med 2011;270:327-38.
11. Krumina A, Vasiljeva G, Ivanovs A, et al. Assessment of value of fatigue severity and symptoms in patients with chronic fatigue syndrome/myalgic encephalomyelitis and fibromyalgia. Br J Med Med Res 2014;4:5866-77.

12. Reeves WC, Lloyd A, Vernon SD, et al. Identification of ambiguities in the 1994 chronic fatigue syndrome research case definition and recommendations for resolution. BMC Health Serv Res 2003;3:25.

13. Arpino C, Carrieri MP, Valesini G, et al. Idiopathic chronic fatigue and chronic fatigue syndrome: a comparison of two case-definitions. Ann Ist Super Sanita 1999;35:435-41.

14. Líndal E, Stefánsson JG, Bergmann S. The prevalence of chronic fatigue syndrome in Iceland - a national comparison by gender drawing on four different criteria. Nord J Psychiatry 2002;56:273-7.

15. Lloyd AR, Wakefield D, Boughton $\mathrm{C}$, et al. What is myalgic encephalomyelitis? Lancet 1988;1:1286-7.

16. Holmes GP, Kaplan JE, Gantz NM, et al. Chronic fatigue syndrome: a working case definition. Ann Intern Med 1988;108:387-9.

17. Ranjith G. Epidemiology of chronic fatigue syndrome. Occup Med 2005;55:13-19.

18. Dinos S, Khoshaba B, Ashby D, et al. A systematic review of chronic fatigue, its syndromes and ethnicity: prevalence, severity, comorbidity and coping. Int $J$ Epidemiol 2009;38:1554-70.

19. Johnston S, Brenu EW, Staines DR, et al. The adoption of chronic fatigue syndrome/myalgic encephalomyelitis case definitions to assess prevalence: a systematic review. Ann Epidemiol 2013;23:371-6

20. Shojania KG, Sampson M, Ansari MT, et al. Updating Systematic Reviews: Agency for Healthcare Research and Quality, 2007. http://www.ncbi.nlm.nih.gov/pubmed/20734512. (accessed 13 Oct 2017).

21. Dowsett EG, Goudsmit E, Macintyre ASC. The National Task Force on Chronic Fatigue Syndrome (CFS), Post Viral Fatigue Syndrome (PVFS), Myalgic Encephalomyelitis (ME): Westcare, 1994.

22. Institute of Medicine IOM. Beyond Myalgic Encephalomyelitis/ Chronic Fatigue Syndrome: Redefining an IIIness: Natl Acad Press, 2015.

23. Munn Z, Moola S, Lisy K, et al. Methodological guidance for systematic reviews of observational epidemiological studies reporting prevalence and cumulative incidence data. Int $J$ Evid Based Healthc 2015;13:147-53.

24. von Elm E, Altman DG, Egger M, et al. The Strengthening the Reporting of Observational Studies in Epidemiology (STROBE) statement: guidelines for reporting observational studies. Lancet 2007;4:e296.

25. Higgins JP, Thompson SG, Deeks JJ, et al. Measuring inconsistency in meta-analyses. BMJ 2003;327:557-60.

26. Moher D, Liberati A, Tetzlaff J, et al. Preferred reporting items for systematic reviews and meta-analyses: the PRISMA statement. BMJ 2009;339:b2535. 